\title{
PAPR REDUCTION TECHNIQUES AND IMAGE QUALITY ASSESSMENTIN IMAGE BASED MMS VLC SYSTEM
}

\author{
Kiranmai Babburu ${ }^{1}$, Kapila Gurucharan ${ }^{2}$, S S Kiran ${ }^{3}$, P Srujana ${ }^{4}$ \\ 1,2,3,4 Electronics and Communication Engineering, Lendi Institute of Engineering and Technology, India, \\ ${ }^{1}$ kbabburu@gmail.com, ${ }^{2}$ gurucharan91@gmail.com, ${ }^{3}$ sskiran88k@gmail.com, ${ }^{4}$ p.srujana17@gmail.com
}

\begin{abstract}
The radiofrequency method of transmission suffers from the problem of the limited licensed radio frequency spectrum, \& security, wide EMI (electromagnetic interference), high multipath fading \& power consumption for short-range applications. The solution to these problems can be achieved by using optical wireless communication technique called the Visible Light Communication system where the data is sent through regulated emission of light. The transmission of digital image information productively in the present optical wireless communication framework is an uphill task as these systems often suffer from problems like frequency selective fading propagation losses and inter-symbol interferences. In order to conquer such difficulties we hereby propose a robust method to transfer the digital image through a Multi-Carrier Modulation Scheme-MMS. In this paper, an Image-Based Multicarrier modulation VLC system is designed through which is sent a corrupted Digital Image with additive white Gaussian noise and explored the overall execution of the Image based Multicarrier modulation VLC system at distinct SNR values. The paper also addresses the issue of high Peak to Average Power Ratio (PAPR) in Multicarrier modulation and reduced it by using different PAPR reduction techniques. The received image quality of the proposed system is obtained under different signal to noise ratios at different modulation schemes..
\end{abstract}

Keywords: Multicarrier Modulation Scheme; AWGN; BPSK; VLC; PAPR

\section{Introduction}

The wireless technologies help humans to communicate through wireless media from anywhere and any instant of time using electromagnetic waves in open space. The different types of wireless technologies that are used in Radio Frequency band include Bluetooth, Zigbee, and Wi-Fi (Wireless Fidelity). Wireless technology usage has spread widely in the last few years because people need to always stay connected at high speed to the network. Moreover, with the expansion of web technologies, the portion of information sent has been expanded radically. These requirements are met through the use of radio frequency based wireless technologies but in future as the volume of transmitted data increases there is a greater demand to move towards a new wireless technology that can offer Large unlicensed bandwidth.

Optical wireless communication (OWC) is likely to be the new prospect of the future wireless technologies.in these wireless technologies the data is transferred through regulated emission of light. The notable advantage of moving towards OWC is that it provides large unlicensed spectrum which can be utilized by anyone to design and commercialize OWC system. Furthermore, the OWC systems offer larger bandwidth compared to that offered by radio frequency bandwidth.

Visible light communications (VLC) is subset of optical wireless communication technologies. Visible light communication is carried through the visible spectrum whose band is $869 \mathrm{THz}-400 \mathrm{THz}$ which is almost 1600 times wider than the radio bandwidth. The VLC is highly secure as these signals cannot pass through opaque objects thus restricting the signal to a confined area.

The connectivity of the wireless communication network can be greatly enhanced by the use of Visible light communication.

Multicarrier Modulation Scheme has been widely researched in VLC [11] frameworks because of benefits like robustness to selective fading of the frequency and the high spectrum efficiency. Lately, with the advancement of computer and Internet innovation, images are generally used to transfer the data. An image may be defined as a two-dimensional mathematical function $f(x, y)$. The value of $f(x, y)$ at any pair of $(x, y)$ of an image plane provides the intensity of the image at that point. When $\mathrm{f}(\mathrm{x}, \mathrm{y})$ and amplitude levels of $\mathrm{f}$ are all finite, discrete quantities a digital image is formed. The goal is to find out a way through which the digital image can be sent through the effective use of modern transceivers.

Modern transceivers are required to meet the demands such as High capacity and variable bit rate information transmission with high bandwidth efficiency in order to provide quality service to the customer. Conventional single-carrier wireless communication frameworks don't perform well as they are generally impeded by fading and multipath delay spread occurrence. These systems also suffer from extreme fading of 
the signal amplitude and Inter Symbol Interference (ISI) at the receiver side which brings about a high probability of errors and the system's overall performance becomes very poor. Channel coding ad adaptive equalization techniques have been extensively used to provide a quick fix to the above problems. Nonetheless, as a result of the inherent delay in the coding and equalization process and the huge value of the hardware, it is quite strenuous to use these approaches in systems operating at large bit rates. A multi-carrier system like Orthogonal Frequency Division Multiplexing technique can be used as a substitute to solve the above problems. This technique has gained popularity in recent times as it is a bandwidth competent technique for wireless communication.

The fundamental thought of these frameworks is that the entire data transfer capacity is separated into many more modest subgroups simultaneously by safeguarding Orthogonality between the groups by utilizing Fast Fourier Transform (FFT) and it's inverse (IFFT). Furthermore, it has the advantages such as high spectral efficiency, straightforward execution, solid multipath resistance, vigorous against thin band co-channel interference and channel fading, and so on. The multicarrier modulation method is additionally appended with the cyclic prefix (CP) to repudiate the impact of the delay. Image Quality metric plays an important role [13] in image processing applications. These metrics are useful in monitoring the quality of the image in quality control systems, useful in benchmarking image processing algorithms and these metrics can be embedded into real time image processing system to optimize the algorithms and parameter settings. Peak Signalto-Nose Ratio (PSNR) and Mean Squared Error (MSE) are the most widely used objective image quality distortion metrics, but they are widely criticized as well, for not correlating with perceived quality measurement. In most of the cases, there is no access to the original image the image quality at the receiver cannot be estimated properly. This problem can be resolved with the use of perception image quality evaluator [12] which determines the image quality without any statistical analysis.

The organization of the entire report is as follows. In section1 we discussed about the Introduction and section 2 Literature Review. This was followed by section 3 where we discuss about our proposed system. Section 4 deals with results and Section 5 concludes the highlighting important points and also future work by setting new research direction

\section{Literature Review}

In this section, we will discuss an overview of our research work carried out in the field of wireless communication with the use of Multicarrier modulation an attempt has also been made to introduce the concept of Multicarrier modulation, its advantages and applications through the already completed studies in the concerned subject area. T. Esmailian, et.al [1] in their paper "Characteristics of In building Power lines at high frequencies and their channel capacity" stated that Communication systems were designed to establish a connecting link between a source to one or more destinations. They recognized that in the first half of 20th century, during the evolution of analog communication and in second half during evolution of digital. C. Eklund, et.al [2] in their paper titled "A technical overview of the Wireless MANTM air interface for broadband wireless access" stated the difference between Multicarrier modulation and other digital communication techniques. They concluded that Multicarrier modulation, one of the best methods to provide high data rate transmission through limited bandwidth. A.R.S.. Bahai, et.al [3] in their paper titled "Multi-carrier digital communications: theory and application of Multicarrier modulation" stated Multicarrier modulation allows digital data transmission over channel for wideband digital communication with efficient signaling scheme having harsh characteristics. They also stated that Multicarrier modulation is mainly considered as a multiplexing technique rather than modulation technique.

DanRaphaeli [4] in their paper titled "A Comparison between OFDM, Single Carrier, and Spread Spectrum for high Data Rate" compared the advantages of multicarrier communication technique with single carrier system. They also stated the fact that even for a small interference in a single carrier system will collapse the entire system, whereas in multi carrier system there will be a minute effect on subcarriers. Error Correction Coding (ECC) method used to recover the effected subcarriers. B.R. Saltzberg [5] in their paper titled "Performance of an efficient parallel data transmission system" stated the early application of using the Multicarrier modulation systems in military communications. "Some of these military based researches was completed by Bello [6], Zimmemann [7], powers and Zimmermann[8], Chang and Gibby[9]."

Jianhua Liu [10] in their paper "System design and signal processing for ofdm -based wireless communication systems" stated the reason behind the application of Multicarrier modulation in military communication systems is due to the fact that it has a great capacity to fight against the multipath fading using Inter Symbol Interference.

T. Komine and M. Nakagawa, [11] in their paper "Fundamental analysis for visible light communication system using LED light", gave insights about the optical wireless communication technology and utilization of White LED light for indoor visible light communication. N. Venkatanath, D. Praneeth, Bh. M. Chandrasekhar, S. S. Channappayya, and S. S. Medasani [12] "Blind Image Quality Evaluation Using Perception Based Features, presented a completely blind NRQuality Evaluation Metric that uses cues from human visual system to address the problem of blind image quality assessment', Zhou Wang and Alan C. Bovik [13], in their paper "why is image quality assessment so difficult?" presented the need for image quality assessment tools and their role in image processing applications. 


\section{Proposed System}

Multi Carrier Modulation Scheme uses modulation and multiplexing technology; it is often referred as a Multi-Tone, Multi carrier system. In this transmitted data is spread over more number of carriers, and by proper choosing of frequency spacing among them, they will become orthogonal to each other. Orthogonality between the sub carriers ensures that they will be separated easily at the receiver thereby giving us superior spectral efficiency. In Multicarrier modulation the bandwidth of signal is divided into number of narrow sub bands so that the frequency response of each individual sub bands is relatively flat, so that the need for equalizer is not required but whereas in single carrier system it is required In the proposed system, the input image is converted into Multicarrier modulation data using symbols in spectral space using M-PSK ( $M=2,416$ \& 256), QAM, etc., and now it convert into time domain from spectral by using Inverse Fast Fourier Transform (IFFT).

The Multicarrier modulation signal can be expressed mathematically by the following equation:

$\mathrm{Y}(\mathrm{t})=\sum_{\mathrm{l}=0}^{\mathrm{M}-1} \mathrm{Y}_{\mathrm{l}} \mathrm{e}^{\mathrm{i} 2 \mathrm{rl} \mathrm{t} / \mathrm{T}}, \quad 0 \leq \mathrm{t}<\mathrm{T}$

(Eq 3.1)

The above equation is also the discrete Fourier transform. where $\mathrm{Y}_{1}$ are image data symbols which is the sequence of complex numbers representing BPSK,QPSK,16 PSK,256 PSK,4,8,\&16QAM base band symbols, M represents number of sub-carriers and $\mathrm{T}$ is Multicarrier modulation symbol line. For each symbol period, the subcarrier spacing makes it as an orthogonal. The sequence of multicarrier modulation is given as follows:

$\mathrm{S}(\mathrm{t})=\sum_{\mathrm{l}=-\infty}^{\infty} \mathrm{Y}(\mathrm{t}-\mathrm{IT})$.

In order to reduce the inter symbol interference before the Multi Carrier Modulation Scheme (MMS) block a guard having an interval of length is inserted. During this interval a cyclic prefix is transmitted which is in the form of

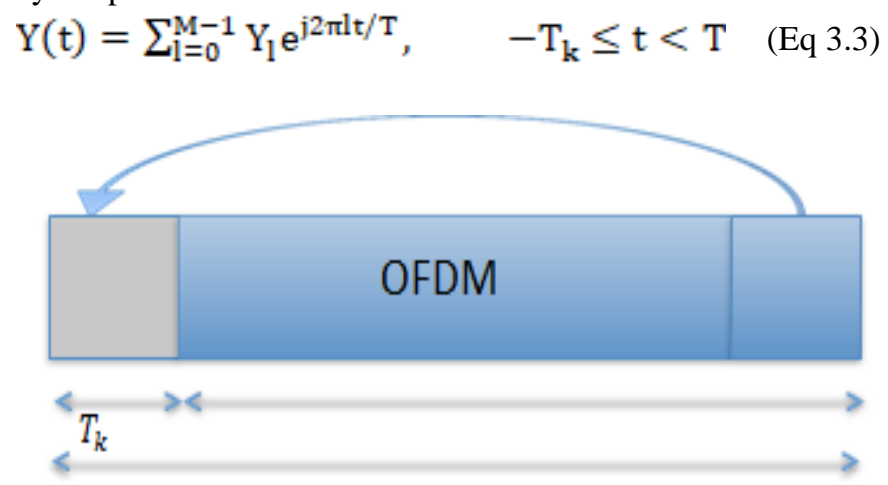

Fig 3.1: Multicarrier modulation Symbol with CP OF Length The Fast Fourier Transform (FFT) and Inverse Fast Fourier Transform (IFFT) which are computationally efficient techniques for implementation of Discrete Fourier Transform and its inverse are the main modulation and demodulation techniques used in the multicarrier modulation. In the existing method a gray scale image is transmitted by using single modulation technique from transmitter to receiver through an ideal channel without noise.

This proposal is to improve image transmission by using multicarrier modulation system over AWGN channel. In the proposed methodology, the 3-dimensions of color image is given as the input to the multicarrier modulation system in order to study and explore the overall performance of the multicarrier modulation system over AWGN channel for different SNR. The evaluation of the system is done by using different modulation techniques like BPSK,QPSK,16PSK,256PSK,4-QAM, 8QAM, 16 QAM using MATLAB and results are displayed.

The proposed system can be summarized as follows:

Step1: A Color Image is taken as input, image quality assessment score is computed and it is converted into digital data.

Step2: Different Digital Modulation techniques are performed on a data like $\mathrm{M}-\mathrm{PSK}(\mathrm{M}=2,4,16,256)$, QAM $(4,16)$

Step3: IFFT is performed on the data \& sent through VLC system

Step4: The time domain signal is transmitted in the error prone VLC link

Step5: FFT is computed

Step6: Demodulation

Step7: Recovered Image \& compute image quality assessment score

Step8: calculation of BER and SNR.

\subsection{System Design}

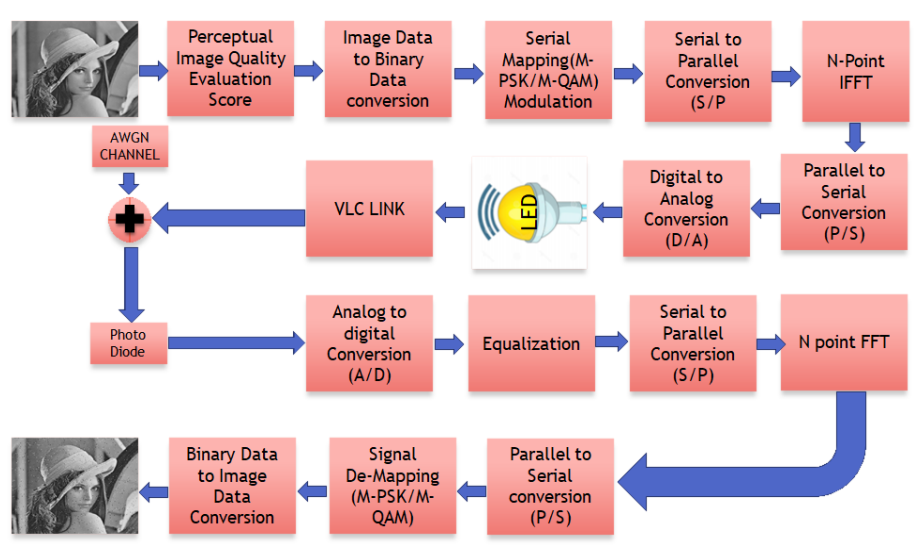

Fig 3.2: Block Diagram of Proposed System The Input Image chosen for the evaluation of the proposed system i.e. Digital Data Transmission over AWGN Channel using multicarrier modulation is "lena.tif" as shown in fig 3.2. The MATLAB data base consists of these by default images which can use by researches. We can access by name 'Lena'. The image is available in the format of .JPG having size $256 \mathrm{X}$ $256 \times 3$.so total number of pixels is $1,96,608$. The value of each pixel is in the form of unsigned integer format. Now the 
image is converted into double format and reshapes the data to change from matrix representation $(256 \times 256 \times 3)$ into Vector representation $(1 \times 1,96,608)$ that means we have converted 3D image into 1D signal. Now this signal is transmitted. In case if we use the Binary Phase shift keying modulation scheme, we need to convert the 1D image data into binary data (two symbols i.e., 0, 1).In case if we use the QPSK modulation scheme, we convert 1D image into binary data(majorly four symbols they are 00,01,10,11 0,1,2,3 respectively). In case if we use 16PSK modulation schemes, then we convert $1 \mathrm{D}$ image into binary data having 16 symbols (0000 to 1111).In case if we use 256 PSK modulation scheme, then we convert 1D image into binary data (256 symbols i.e.00000000 to11111111)

The above data is converted into parallel form by using $\mathrm{s} / \mathrm{p}$ (serial to parallel) converter in order to put the data on multiple subcarriers which are modulated above mentioned modulation schemes which is followed by Inverse Fourier Transform and the signal is converted into serial by using $\mathrm{p} / \mathrm{s}$ (parallel to serial) converter. This data is sent to Digital to analog converter and sent through the LED as light pulses. At this point, Image based multicarrier modulation VLC transmitter section comes to an end. This data after the transmitter block is passed through a VLC Channel corrupted by additive white Gaussian noise. At the receiver section inverse operations are performed which includes recovering the data using photodiode, estimating the symbol timing, removal of cyclic prefix, now the signal is passed through Fast Fourier Transform which recovers the subcarriers according to the modulation technique employed. After FFT is performed the data is converted to serial by using $\mathrm{p} / \mathrm{s}$ (parallel to serial) converter. The signal is de mapped depending upon the modulation scheme employed this process is called demodulation. After demodulation, post-processing is performed to convert the digital data obtained into a digital image. This obtained image is called the Recovered image. The bit error rate at different SNR is found for different modulation schemes and a graph is plotted to evaluate the performance of system under AWGN Noise.

\subsection{PAPR Reduction Techniques for Proposed Image Based MMS}

In multicarrier modulation framework, the main drawback is high peak to average power proportion (PAPR).It is mainly caused due to IFFT where information symbols across sub carriers deliver high power esteem. The peak to average power proportion of discrete time signal is characterized as

PAPR $=\frac{P_{\text {peak }}}{P_{\text {average }}}=\frac{\max _{0 \leq n \leq \mathbb{N}-1}\left[|x(n)|^{2}\right]}{E\left\{\|\left._{x}(n)\right|^{2}\right\}}$

(Eq 3.4)
Where $\mathrm{P}_{\text {peak }}$ indicates the peak power and $\mathrm{P}_{\text {average }}$ indicates the average output power, $\mathrm{E}$ indicates the expected value and $\mathrm{x}(\mathrm{n})$ indicates the transmitted MMS signal.

In this segment, we mostly talk about three important procedures, for example, Amplitude Clipping and separating, Selective mapping and partial transmit sequence for PAPR decrease in image based multicarrier modulation frameworks.

\subsubsection{Selective Mapping (SLM)}

In Selective Mapping (SLM) the whole game plan of information picture are stacked into the subcarrier and turn in consecutive and equivalent after, the data block are structure and a while later the most great sign with less PAPR is picked and conveyed. The fundamental idea of this system relies upon the stage revolution progression. The most insignificant PAPR signal will be picked for transmission from different assorted data hinders self-governing.

In this algorithm, the input block $S=[S[0], S[1], S[2], \ldots . S[N-1]]$ is multiplied with $\mathrm{X}$ different phase sequences $P^{x}=\left[P_{0}{ }^{x}, P_{1}{ }^{x}, P_{2}{ }^{x}, \ldots \ldots P_{N-1}{ }^{x}\right]^{T}$ where $P_{y}^{x}=e^{j} \emptyset_{y}^{x} \quad$ and $\quad \emptyset_{y}^{x} \in[0,2 \pi)$ for $y=0,1,2, \ldots . N-1$ and $x=1,2, \ldots . X$ which produce a modified data block $S^{x}=\left[S^{x}[1], S^{x}[2], \ldots . . S^{x}[N-1]\right]^{T} . \quad$ IFFT of $\mathrm{X}$ independent sequences $\left\{S^{x}[y]\right\}$ are taken to produce the sequencess $s^{x}=\left[s^{x}[0], s^{x}[1], \ldots \ldots s^{x}[N-1]\right]^{T}$, among which $\stackrel{s}{s}=s^{\tilde{x}}$ with the lowest PAPR selected [16] for transmission as shown below

$\check{x}=\operatorname{argmin}_{x=1,2,3 \ldots x}\left(\max _{x=1_{2}, 2,3 \ldots x}\left|s^{x}[n]\right|\right)$

(Eq 3.5)

\subsubsection{Partial Transmit Sequence (PTS):}

The Partial Transmit Sequence (PTS) method segments an information square of $\mathrm{N}$ images into $\mathrm{V}$ disjoint sub Squares as $S=\left[S^{0}, S^{1}, \ldots . S^{v-1}\right]^{T}$

(Eq 3.6)

Where $S^{i}$ are the sub-squares that are successively found and furthermore are of equivalent size. Dissimilar to the SLM strategy in which scrambling is applied to all subcarriers, scrambling (rotating its phase freely) is applied to each subsquare [14] in the PTS method.

At that point, each segmented sub block is multiplied by a relating complex phase factor

$b^{v}=e^{j \phi v}, v=1,2, \ldots V$

Hence taking its IFFT to yield

$s=\operatorname{IFFT}\left\{\sum_{v=1}^{V} b^{v} \cdot S^{v}\right\}=\sum_{v=1}^{V} b^{v} . \operatorname{IFFT}\left\{S^{v}\right\}=$ $\sum_{v=1}^{V} b^{v} \cdot s^{v}$

(Eq 3.8) 
Where $\left\{s^{v}\right\}$ is referred to as a partial transmit sequence (PTS).

PTS algorithm:

1. Partition the information data block into $\mathrm{V}$ sub squares as in Equation (3.5).

2. Set all the stage factors bv $1 / 41$ for $v 1 / 41: \mathrm{V}$, discover PAPR of Equation (3.7), and set it as PAPR_min.

3. Set $v=2$

4. Find PAPR of Equation (3.4) with

5. If PAPR> PAPR_min, switch back to 1 . Something else, update PAPR_min=PAPR.

6. If $\mathrm{v}<\mathrm{V}$, increase $\mathrm{v}$ by one and return to Step 4.Otherwise, leave this cycle with the arrangement of ideal stage factors, b.

\subsection{Image Quality Assessment}

In the proposed block diagram, the image quality is assessed before transmission and at the receiver through the perception image quality evaluator (PIQE) algorithm. PIQE ascertains the no-reference quality score for an image through square shrewd bending assessment, utilizing these means:

1. Compute the Mean Subtracted Contrast Normalized (MSCN) coefficient for each pixel in the image using the estimation proposed by N. Venkatanath and others [1].

2. Divide the data picture into non covering squares of size 16-by-16.

3. Identify high spatially unique squares reliant on the fluctuation of the MSCN coefficients.

4. Generate development Mask using the recognized high spatially powerful squares.

5. In each square, evaluate contortion in light of obstructing curios and clamor using the MSCN coefficients.

6. Use edge measures to portray the squares as mutilated squares with impeding antiques, misshaped squares with Gaussian clamor, and undistorted squares.

7. Generate detectable Artifacts Mask from the deformed squares with blocking collectibles and commotion Mask from the mutilated squares with Gaussian clamor.

8. Compute the PIQE score for the information picture as the mean of scores in the mutilated squares.

The quality size of the picture dependent on its PIQE score is given in this table 3.1

\begin{tabular}{|l|l|}
\hline Quality Scale & Score Range \\
\hline Excellent & {$[0,20]$} \\
\hline Good & {$[21,35]$} \\
\hline Poor & {$[51,80]$} \\
\hline Bad & {$[81,100]$} \\
\hline
\end{tabular}

Table 3.1: Image Quality Assessment Score.

\section{Results and Analysis}

In proposed image based multicarrier modulation VLC system, the performance of the system is evaluated by sending the standard Lena Image is taken as input image through the system. This image is converted to bit stream and modulated using different modulation schemes such as QPSK, 16PSK,256-PSK, added with additive white Gaussian noise at different SNR and sent over the AWGN channel with the help of Visible Light Communication. The results presented discuss about the PAPR received at the receiver after modulation (PAPR original) and also the performance of PAPR reduction methods such as Selective Mapping and Partial Transmit Sequence at different SNR and different modulation schemes

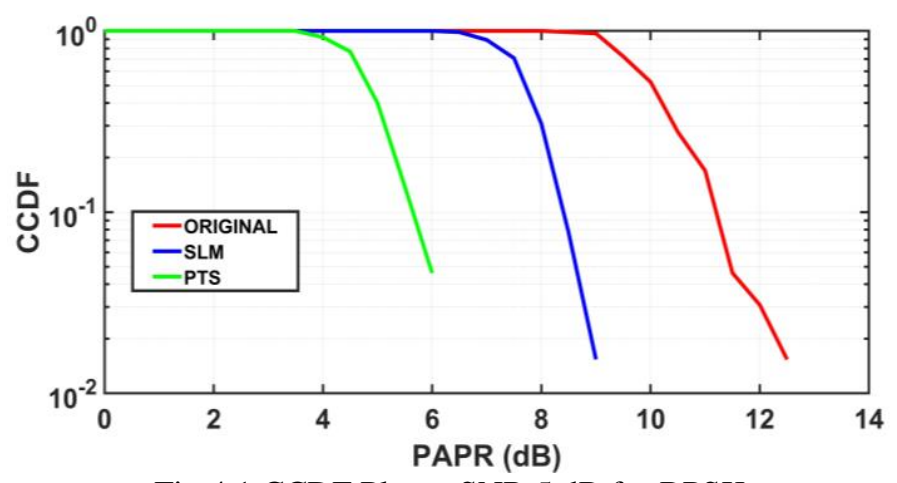

.Fig 4.1 CCDF Plot at SNR $5 \mathrm{~dB}$ for BPSK

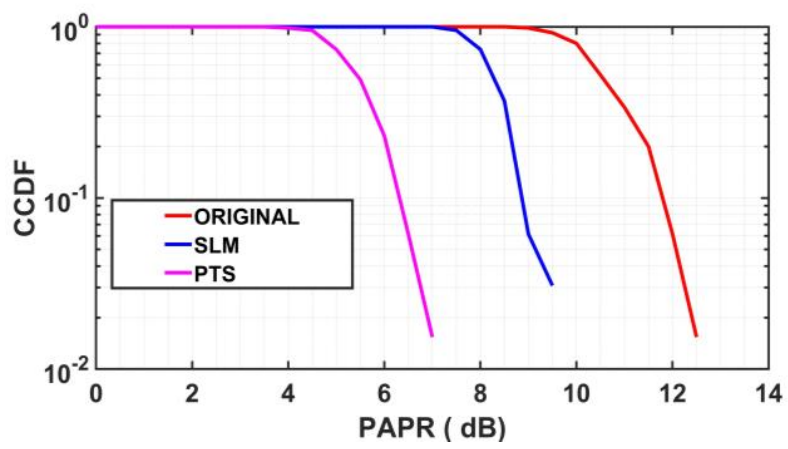

Fig 4.2 CCDF Plot at SNR 5dB for QPSK

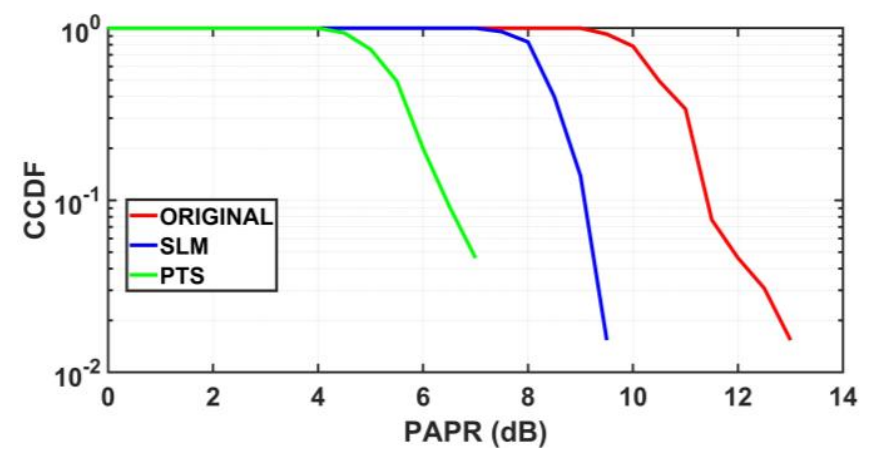

Fig 4.3 CCDF Plot at SNR 5dB for 16-PSK. 


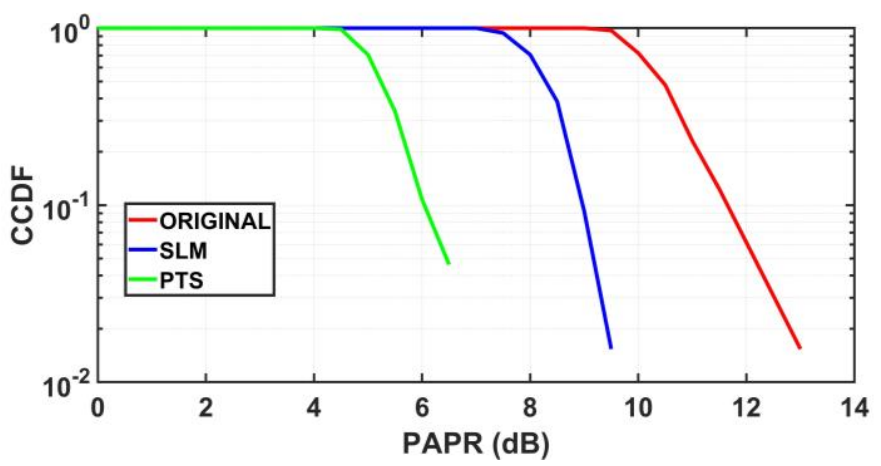

Fig 4.4 CCDF Plot at SNR 5dB for 256 PSK.

The above figure 4.1 to 4.4 illustrates the CCDF Plot for different modulation schemes at SNR $5 \mathrm{~dB}$. The performance of QPSK CCDF plot at SNR $5 \mathrm{~dB}$ is better than all modulation schemes.
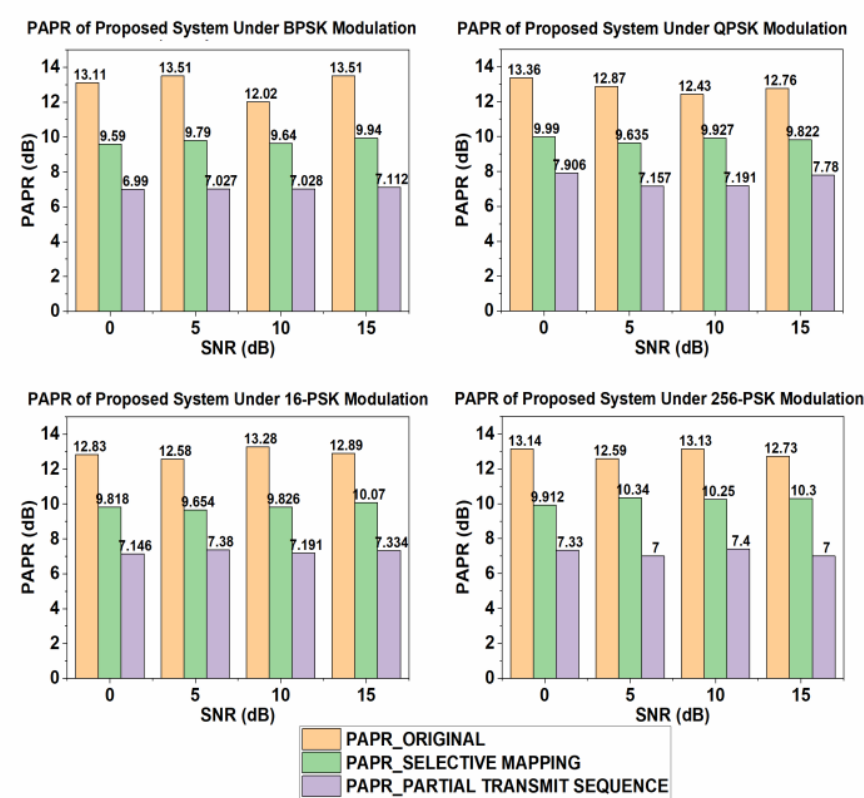

Fig 4.5 Comparison of PAPR (dB) for different modulation techniques at different SNR (dB).

The figure 4.5 illustrates the comparison of PAPR $(\mathrm{dB})$ for different modulation techniques at different SNR (dB).The overall performance of QPSK is better than all other modulation techniques at different Signal to Noise Ratios. The figure 4.5 shows the results of comparison of PAPR reduction techniques like Selective mapping (SLM) and Partial Transmit Sequence (PTS). The Partial Transmit Sequence (PTS) PAPR Reduction Technique performs better all modulation techniques and all Signals to Noise Ratios.

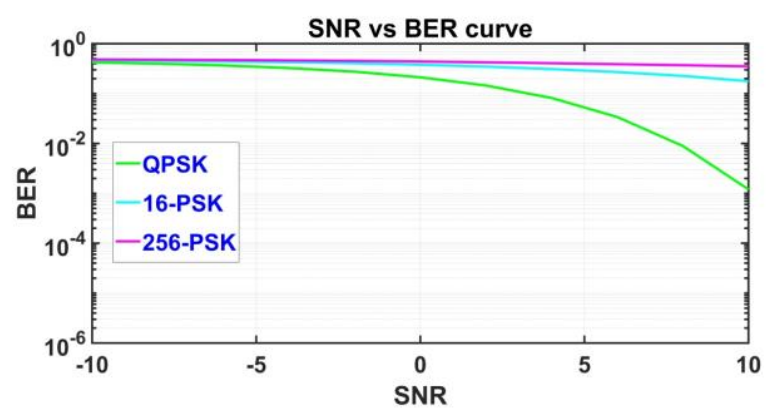

Fig 4.6 Performance of Different Modulation Schemes at Different SNR

The above figure 4.6 illustrates the performance of image based MMS at different Signal to noise ratios. The performance of QPSK is better when compared with other modulation schemes. At SNR $5 \mathrm{~dB}$ a better reception is possible for QPSK when compared with other modulation schemes.

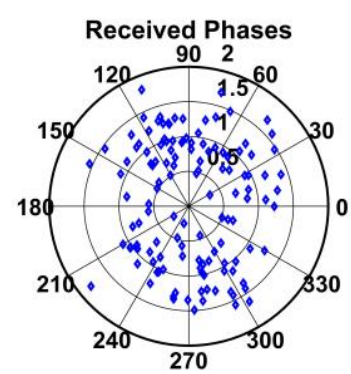

Fig 4.7 Received Phases at SNR $5 \mathrm{~dB}$ for BPSK modulation scheme.

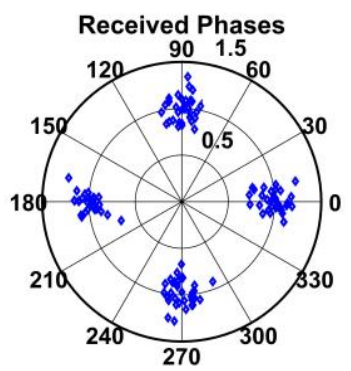

Fig 4.8 Received Phases at SNR $5 \mathrm{~dB}$ for QPSK modulation scheme.

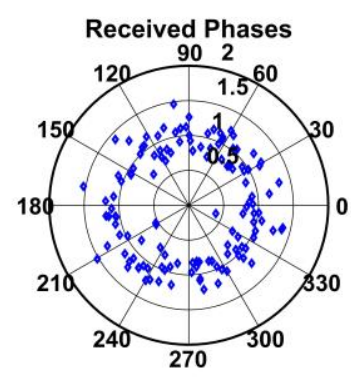

Fig 4.9 Received Phases at SNR 5 dB for 16PSK modulation scheme. 


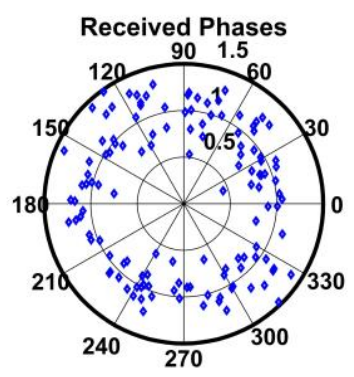

Fig 4.10 Received Phases at SNR 5 dB for 256 PSK modulation scheme.

The above figure 4.7 to 4.10 depicts the received phases at SNR $5 \mathrm{~dB}$ for different modulation schemes employed the Received Phases for QPSK at SNR 5dB shows better response than other modulation schemes at same SNR.

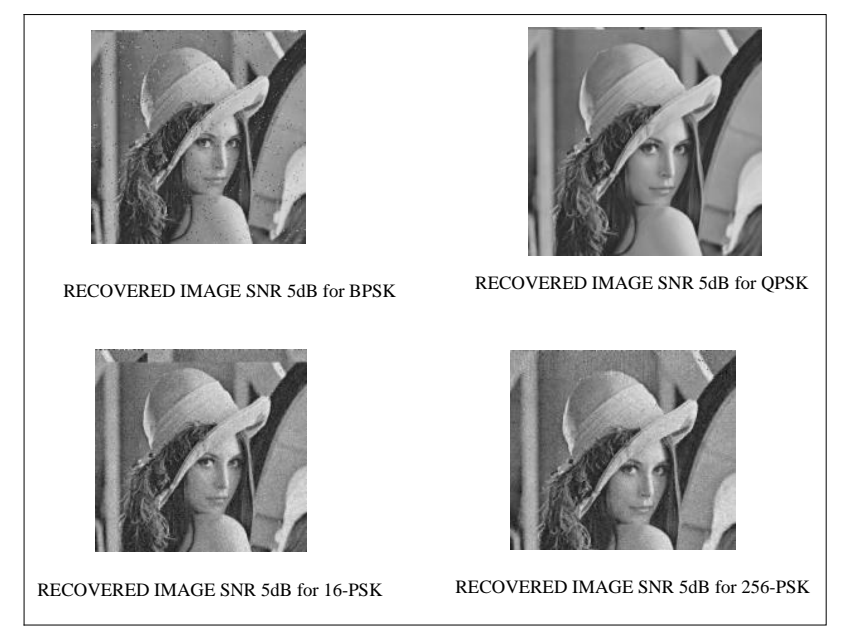

Fig 4.11 Recovered Images at SNR 5dB for different modulation schemes.

The above figure 4.11 shows the recovered images at SNR $5 \mathrm{~dB}$ for different modulations schemes. The performance of image based MMS is better when QPSK modulation scheme is employed.

It is observed that the perceptual image quality evaluator score for input image is 31.753755 (Good) and the recovered image at the receiver of Proposed system has perceptual Image Quality Evaluator score 36.347990 (Fair). It can be observed from figure that the original PAPR is computed to be $12.5 \mathrm{~dB}$, with Amplitude Clipping \& filtering the PAPR is reduced to $10.48 \mathrm{~dB}$, with Selective mapping the PAPR is reduced to 9.7 $\mathrm{dB}$ and with Partial Transmit Sequence the PAPR is reduced to $7.08 \mathrm{db}$.

\section{Conclusion}

The Proposed system has been implemented for different modulation techniques for transmission of image through AWGN channel. From this proposed system, the quality of image is high for higher SNR values for all types of modulation techniques, whereas the quality of image is poor for lower SNR values due to AWGN noise.

The BER vs. SNR curves at several modulation schemes are simulated using MATLAB and the possible SNR at which the original image can be recovered for each of these digital modulation techniques is also presented. The high Peak to Average Power Ratio (PAPR) is observed in Multicarrier Modulation Scheme (MMS) as $12.5 \mathrm{~dB}$ which is reduced by using different PAPR reduction techniques like Amplitude Clipping, Selective mapping (SLM) and Partial Transmit Sequence (PTS), the comparative results of these techniques are presented. From the results it is observed that the Partial Transmit Sequence (PTS) PAPR reduction technique obtained $7.08 \mathrm{~dB}$ and this is better than other techniques. The image quality both at the transmitter and receiver sections of image based MMS VLC System has been evaluated using Perception based Image Quality Evaluator (PIQE) is found to be 31.753755 and 36.347990 respectively.

\section{References}

[1] Esmailian, Tooraj \& Kschischang, Frank \& Gulak,P.In-building power lines as high-speed communicationchannels:Channelcharacterizationan datestchannelensemble.Int.J.Communication Systems.

[2] C.Eklund.IEEEStandard802.16:ATechnicalOvervie woftheWirelessMANAirInterfaceforBroadbandWir elessAccess,IEEECommun.Mag,volume40,pp. 98107.

[3] A.R.S. Bahai, B. R. Saltzberg and M. Ergen. Multicarrierdigitalcommunications:theory andapplicationofOFDM.2ndedition:Springer Science. pp. $55-68$.

[4] DanRaphaeliandEvgeni Bassin.AComparison BetweenOFDM,SingleCarrier,andSpreadSpectrumf orhighDataRatePLC.ISPLC,pp.162 - 168.

[5] B.R.Saltzberg,Performanceofanefficientparalleldata transmissionsystem,IEEETransactionson Communication, Volume15,pp.805-813.

[6] P. Bello. Selective fading limitations of the KATHRYN modem and some system design considerations, IEEETransactionsonCommunication sTechnology,volume13,pp.320-333.

[7] M.ZimmermannandA.Kirsch.TheAN/GSC10/KAT HRYN/variableratedatamodemforHFradio,IEEETra nsactionsonCommunicationTechnology,volume15,p p. $197-204$

[8] E.PowersandM.Zimmermann.Adigitalimplementati onofamultichanneldatamodem.Proceedings of the IEEE International Conference on Communication.

[9] R. Chang and R. Gibby.A theoreticalstudyof performanceofanorthogonalmultiplexingdatatransmi 
ssionscheme,IEEETransactionson

CommunicationTechnology,volume16,pp.529-540.

[10] Jianhualiu.Systemdesignand signal processing forOFDM-basedwirelesscommunication systems.University of Florida ,PhD thesis.

[11] T.Komine;M.Nakagawa,Fundamentalanalysis forvisible-lightcommunicationsystemusing LEDlights,IEEETransactionsonConsumerElectronic s, Volume50,pp 100 - 107

[12] N.Venkatanath,D.Praneeth,Bh.M.Chandrasekhar,S. S.Channappayya,andS.S.Medasani.BlindImageQual ityEvaluationUsingPerceptionBasedFeatures, In Proceedings of the 21st National Conference on Communications (NCC). Piscataway, NJ: IEEE, 2015.

[13] ZhouWang,AlanC.Bovik,LigangLu,Whyisimagequa lityassessmentsodifficult?,IEEE,InternationalConfer enceonAcoustics,Speech, andSignal Processing.

[14] Muller,S.H.OFDMwithreducedpeaktoaveragepower ratiobymultiplesignalrepresentation.In Annals of Telecommunication, volume 52,pp. 58-67.

[15] vanNee,R.anddeWild,A.Reducingthepeaktoaveragepowerratio of OFDM. IEEE VTC'98, volume.3, pp. 18-21.

[16] Bauml,R.W.,Fischer,R.F.H.,andHuber,J.B.Reducing thepeaktoaveragepowerratioofMulticarriermodulati onbyselectivemapping.Electron.Letter.volume32,pp . 2056-2057.

[17] DavideMattera,MarioTanda,Dataaidedsynchronizati onforOFDM/OQAMsystems,SignalProcessing,Volu me92,pp.2284-2292.

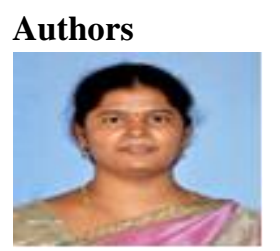

Dr. B Kiranmai working as Professor Department of ECE in Lendi Institute of Engineering and Technology. Qualified PhD in Radar Signal Processing, Area of Research interest is "Radar Systems, Signal Processing, Massive MIMO Systems and Internet of things".

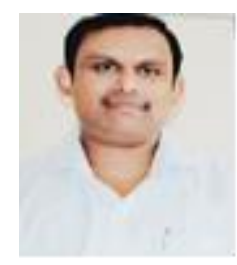

Kapila Guru Charan working as Assistant Professor Department of ECE in Sanketika Institute of Technology and Management, Qualified M.Tech in Digital Systems and Signal Processing, Area of Research interests is 'Embedded Systems, VLSI and Signal Processing.

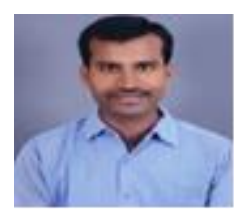

S S Kiran working as Assistant Professor, Department of ECE in Lendi Institute of Engineering and Technology, Qualified M.Tech in VLSI Design, Area of Research interests is "Embedded Systems-ARM Based Controllers, IoT, LOW Power VLSI, Massive MMO Systems and Hybrid Electric Vehicles".

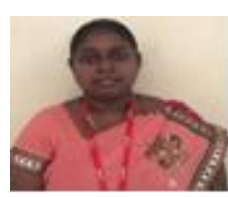

P Srujana working as Assistant Professor, Department of ECE in Lendi Institute of Engineering and Technology, Qualified M.Tech in Communication Engineering and Signal Processing, Area of Research interests is "Embedded Systems", Image Processing, and IoT 